\title{
Crystallisation within intermediate amorphous phases determines the polycrystallinity of nanoparticles from co-precipitation
}

Alexy P. Freitas, ${ }^{\dagger, \ddagger}$ Raj Kumar Ramamoorthy, $^{\dagger}$ Maxime Durelle, ${ }^{\dagger}$ Eric Larquet, ${ }^{\ddagger}$ Isabelle Maurin ${ }^{\ddagger}$ Fabienne Testard, ${ }^{\dagger}$ Corinne Chevallard, ${ }^{\dagger}$ Thierry Gacoin, ${ }^{\ddagger}$ and David Carrière*,†

$\dagger$ †niversité Paris-Saclay, CNRS, CEA, NIMBE, LIONS, 91191, Gif-sur-Yvette, France $\ddagger$ Laboratoire de Physique de la Matière Condensée (PMC), CNRS, Ecole Polytechnique, Institut Polytechnique de Paris, 91120 Palaiseau, France

E-mail: david.carriere@cea.fr

Phone: +33 (0)1 69085489

\begin{abstract}
Intense research on nanocrystals synthesized in solution is motivated by their original physical properties, determined by their sizes and shapes at various scales. However, the mastery of the syntheses is limited by our understanding of crystallisation, challenged by the now well-established prevalence of non-crystalline intermediates. In particular, the impact of such intermediates on the final sizes and c rystal quality remains unclear because characterization of their evolution at nanometer and millisecond scales with non-perturbative analyses has remained a challenge. Here, we evidence from in situ X-ray scattering that nucleation and growth of YVO4:Eu nanocrystals is spatially restrained within amorphous, nanometer-scaled intermediates. The reactivity
\end{abstract}


and size of these amorphous intermediates determine (i) the mono vs polycrystalline character of final crystals, and (ii) the size of final cr ystals. This implies that designing amorphous intermediates themselves, that form in less than $6 \mathrm{~ms}$, is one of the keys towards controlled bottom-up syntheses of optimized nanoparticles.

Nanocrystals synthesis by co-precipitation in water at room temperature and pressure is prevalent in living organisms (formation of spikes and shells) and appealing for industrial processes due to their low environment and energetic footprint. In co-precipitation processes, "classical" visions such as the LaMer model state that the particle size is governed by combination of crystal nucleation rate and reactant depletion: if the nucleation rate is high, most reactants are consumed in the nucleation step and little to no growth is expected, and vice versa. ${ }^{1}$ With this point of view, modulating the nucleation rate by tuning synthesis parameters such as precursor or ligand chemistry, or initial supersaturation should lead to a fine control of the n anocrystal size.

However, the link between the synthesis parameters and the final c rystals sizes is now questioned, because the classical description of a direct transition from the ions to the nascent crystals (classical nucleation) is nowadays amply questioned by experimental evidence: an increasing number of co-precipitation processes are known to follow a so-called "non-classical" crystallisation mechanism that involve non-crystalline intermediate states, such as oligomers, reactant-rich droplets, or amorphous particles. ${ }^{2-15}$ A major challenge of the field is thus to find out the relationship between the structure of the intermediates and that of the final particles. In particular for nanocrystals, the final structural parameters a re p resumably more sensitive to the details of nucleation process, as the typical critical size of nucleation is usually in the nanometer range. ${ }^{16}$ The key to actively controlling nanocrystal size may therefore possibly lie in these intermediate states, which are not considered in classical nucleation theories (CNT) - including the LaMer model. 
Here, we determine how amorphous transient particles, from which nanocrystals nucleate, determine the final microstructure of $n$ anocrystals, i .e. their inner m orphology, porosity, or mono/polycrystalline character down to the nanometer scale. The microstructure of nanoparticles is of practical interest since it impacts significantly the properties of the nanomaterials. For instance, the luminescence properties of YVO4:Eu nanocrystals (yttrium vanadate doped with europium) depend on both the crystal quality and the specific area, so a compromise between a defectless nanomaterial and a fragmented nanomaterial has to be found. ${ }^{17,18}$ The synthesis by co-precipitation of $\mathrm{YVO}_{4}: \mathrm{Eu}$ is a good case study, because we found previously an indirect evidence that crystallisation is confined in the amorphous particles, ${ }^{15}$ and found how to produce polycrystals or monocrystals by varying the alkalinity of the co-precipitation medium. ${ }^{19}$

Here, we show that the different microstructures are indeed determined by a combination of nucleation/growth kinetics, and confinement of the r eaction w ithin the precursor amorphous nanoparticles. The synthesis of luminescent $\mathrm{YVO}_{4}: \mathrm{Eu}$ nanocrystals is conducted by co-precipitation in water at ambient temperature and pressure. Two precursors are mixed in stoichiometric proportions: ${ }^{20}$ (i) a solution containing $\mathrm{Y}^{3+}$ and $\mathrm{Eu}^{3+}$ ions and (ii) a high$\mathrm{pH}$ solution of vanadate, prepared by dissolving sodium metavanadate $\mathrm{NaVO}_{3}$ in water and adding $n \geq 2$ molar equivalents of base to favour conversion into orthovanadate $\mathrm{VO}_{4}^{3-}$ :

$$
\mathrm{VO}_{3}^{-}+n \mathrm{OH}^{-} \rightleftharpoons \mathrm{VO}_{4}^{3-}+(n-2) \mathrm{OH}^{-}+\mathrm{H}_{2} \mathrm{O}
$$

It has been reported before that the colloidal suspensions of $\mathrm{YVO}_{4}$ : $\mathrm{Eu}$ nanocrystals are obtained from a precipitate that forms immediately after mixing. ${ }^{15,20}$ Furthermore, the initial $\mathrm{OH}^{-}$amount $n$ is a control parameter to produce either rough, polycrystalline nanoparticles ( $n=2$, YV2 synthesis), or monocrystalline nanoparticles with well-defined facets $(n=3$, YV3 synthesis), as reported in our previous work. ${ }^{19}$ TEM and XRD show that the particles already possess their mono/polycrystalline character after $24 \mathrm{~h}$ of reaction (Figures 1 and 2, 
SI 2), although facetting is incomplete for YV3 nanocrystals. In order to make a first qualitative comparison of the kinetics of crystallisation, we estimated the volume fraction of $\mathrm{YVO}_{4}: \mathrm{Eu}$ nanocrystals from their characteristic red-light emission under UV excitation (see SI 3). ${ }^{15,20,21}$ We observe an increase in luminescence for YV2 that is two orders of magnitude faster than for YV3 (onset at ca. $30 \mathrm{~s}$ vs $5000 \mathrm{~s}$, see SI 3), suggesting the difference in crystallisation kinetics is linked to the difference in polycrystallinity. However, in the YV3 synthesis, an alternative hypothesis could be that polycrystals form at intermediate reaction times and reconstruct into monocrystals at a later stage. The important question is thus to evidence if monocrystals form directly or via polycrystalline intermediate particles, and if the amorphous intermediates dictate the polycrystallinity of the final nanocrystals.

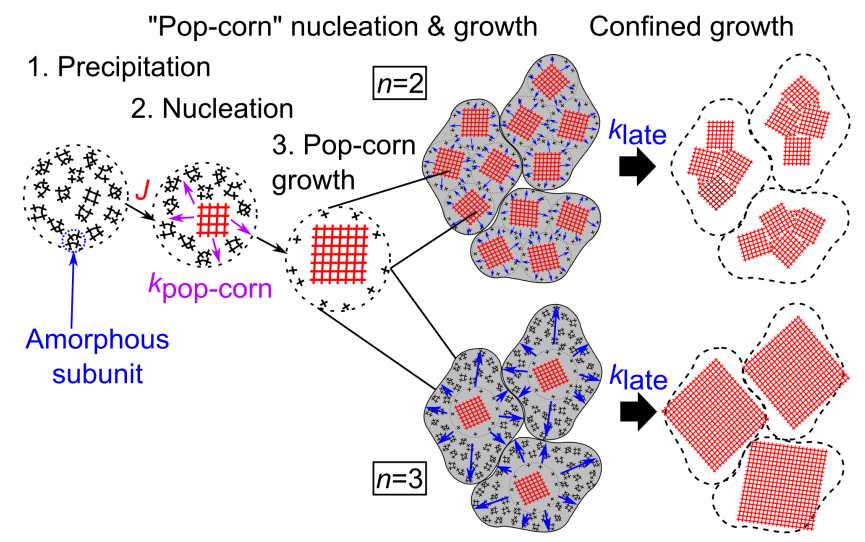

Figure 1: Proposed mechanisms of formation of $\mathrm{YVO}_{4}: \mathrm{Eu}$ nanocrystals as a function of the nominal hydroxyl ratio $n$. In both cases, crystals nucleate from intermediate amorphous particles, grow up to the minimum observable size $k_{\text {pop-corn }}$ and grow further at the rate $k_{\text {late }}$.

To discriminate the direct formation of monocrystals from a subsequent reconstruction, we performed in situ SAXS/WAXS for both syntheses from times as short as 6 ms. SAXS and WAXS patterns evidence that both syntheses involve a transient amorphous aggregate of nanometer-scaled subunits (Fig. 3 and SI 4), consistent with cryoTEM images of YV3 samples quenched prior to crystallization (Fig. 2a-b), and as observed in similar syntheses ${ }^{15}$. At the shortest measured reaction time $(6 \mathrm{~ms})$ and up to at least $20 \mathrm{~s}$, the scattered intensity at low angles is five orders of m agnitude a bove that of water ( SI 4), characteristic of large structures forming upon mixing. Also, at larger angles $\left(q \geq 1 \AA^{-1}\right)$, no Bragg peaks are 

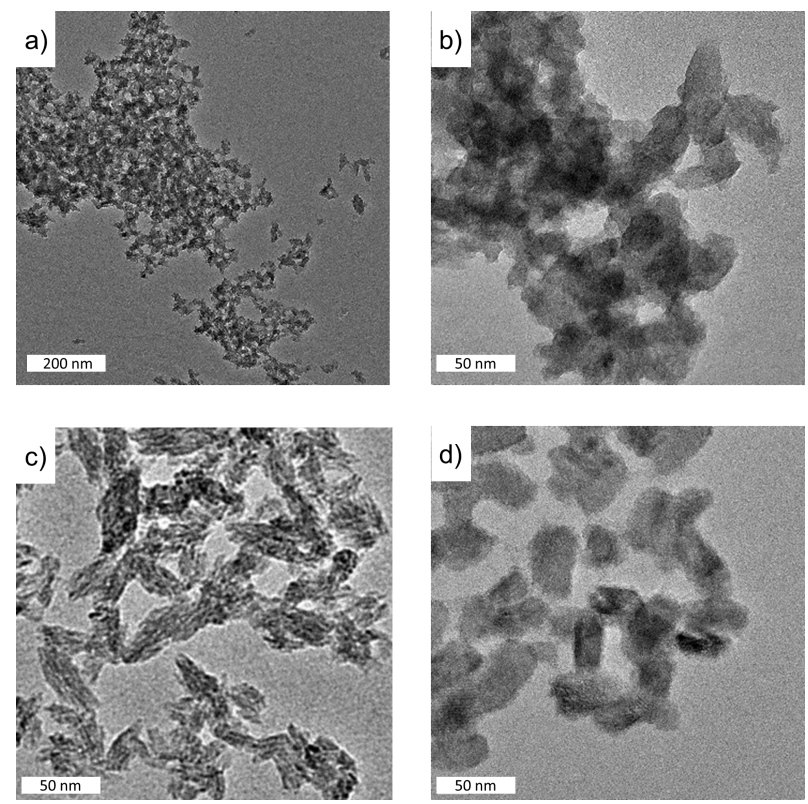

Figure 2: TEM micrographs at $18 \mathrm{~min}$ reaction time for a YV3 synthesis (a and $\mathbf{b})$, and at $24 \mathrm{~h}$ reaction time for the YV2 synthesis $(\mathbf{c})$ and for the YV3 synthesis (d).

observable, but instead a broad correlation peak, evidencing the absence of any small crystals. The amorphous intermediate exhibits three levels of aggregation (see schemes on Fig. 1 and SI 4): subunits with diameter of $c a .1 \mathrm{~nm}$ (inflexion at $q \simeq 0.3 \AA^{-1}$ ) are aggregated into amorphous particles with a diameter of $c a .20 \mathrm{~nm}-25 \mathrm{~nm}$ (inflexion at $q \simeq 8 \times 10^{-2} \AA^{-1}$ ), themselves aggregated into larger fractal structures $\left(I \propto q^{-p}\right.$ with $p \simeq 2.7-3.1$ at low angles). ${ }^{22}$

Contrary to the final nanocrystals, the characteristic sizes of the amorphous intermediate are poorly sensitive to the initial $\mathrm{OH}^{-}$amount $n$. After fitting the Beaucage unified equation commonly used to model fractal aggregation (Eq. S1), ${ }^{23}$ we find that the amorphous particles have a diameter of gyration between ca. $19 \mathrm{~nm}(n=2)$ and $24 \mathrm{~nm}(n=3)$, and their constitutive amorphous subunits with a diameter of gyration of $c a .9 \AA$ in both cases (Table 1). As an indication, for monodisperse, spherical objects it corresponds to respective diameters of $25 \mathrm{~nm}$ (YV2) to $31 \mathrm{~nm}$ (YV3) for the amorphous particles, and of $1.2 \mathrm{~nm}$ for the amorphous subunits (see eq. S2).

At later stages crystallisation occurs, as evidenced by the emergence of Bragg peaks in 

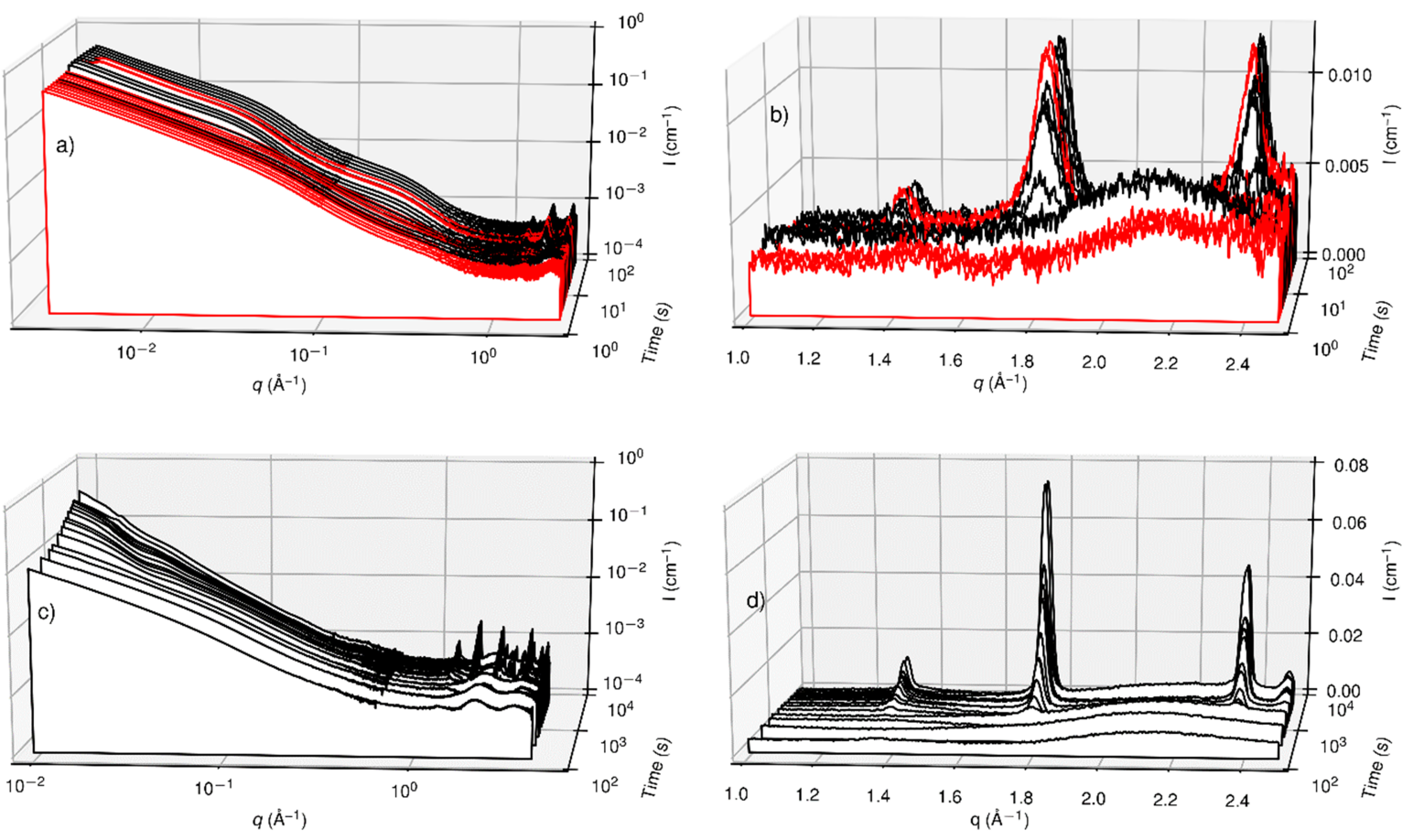

Figure 3: a) Small angle X-ray scattering and b) Wide angle X-ray scattering patterns recorded for a $n=2$ synthesis. (-) recorded with the stopped-flow configuration (total injection flow rate $8 \mathrm{~mL} \mathrm{~s}^{-1}$ ), and (-) recorded with the millifluidic + peristaltic configuration. c) Small angle X-ray scattering and $\mathbf{d}$ ) Wide angle X-ray scattering patterns recorded for a $n=3$ synthesis by uptaking aliquots.

Table 1: Structural parameters for the two syntheses of nanoparticles.

\begin{tabular}{lll}
\hline & \multicolumn{2}{l}{ Initial $\mathrm{OH}^{-}$amount $n$} \\
& 2 & 3 \\
\hline Amorphous primary grain diameter of gyration $(\mathrm{nm})$ & 0.9 & 0.9 \\
Amorphous particle diameter of gyration (nm) & 19 & 24 \\
Crystalline primary grain diameter of gyration (nm) & 2 & 30 \\
Crystalline particle diameter of gyration (nm) & 15 & 30 \\
Crystal coherence lengths (nm) & 10 & 25 \\
\hline
\end{tabular}

the WAXS region (Fig. 3b and d, Fig. 4a). The Bragg peaks appear only after a lag time varying from $c a$. $40 \mathrm{~s}(n=2)$ to $1200 \mathrm{~s}(n=3)$ (Table 2). As discussed earlier, this lag time is also noticed in luminescence measurements (SI 3) and is assigned to the nucleation induction time, i.e. the time needed for oligomers of precursor to overcome the activation barrier and grow up to the detection limit. ${ }^{24}$

During crystallisation, the scattering signal is assumed to be a mixture of purely amor- 
Table 2: Kinetic parameters for the two syntheses of nanoparticles.

\begin{tabular}{lll}
\hline & \multicolumn{2}{c}{ Initial $\mathrm{OH}^{-}$amount $n$} \\
& 2 & 3 \\
\hline Induction time $t(\mathrm{~s})$ & $8-38$ & $1170-1200$ \\
Steady-state nucleation rate $J\left(\mathrm{~m}^{-3} \mathrm{~s}^{-1}\right)$ & $9.8 \times 10^{21}$ & $1.2 \times 10^{15}$ \\
Growth rate during nucleation $k_{\text {pop-corn }}\left(\mathrm{s}^{-1}\right)$ & $0.4-7.8$ & $480-500$ \\
Growth rate after nucleation $k_{\text {late }}\left(\mathrm{s}^{-1}\right)$ & 0.01 & 9.5 \\
\hline Measured number of primary grains per particle & 180 & $\simeq 1$ \\
Predicted number of primary grains per particle $N$ & $32-143$ & $<1$ \\
\hline
\end{tabular}

phous and purely crystalline contributions, which need to be separated. For that purpose, we first computed the total volume fraction of the amorphous phase from the total scattered intensity in the absence of Bragg peaks using the invariant theorem $(Q=$ $\int_{0}^{\infty}\left[I_{\text {model }}(q)-I_{+\infty}\right] q^{2} \mathrm{~d} q$, eq. S1 and S3), which relates the total scattered intensity to the volume fraction and electronic density of the scatterers and states that the total scattered intensity is independent of the scatterer spatial arrangement. This volume fraction was found equal to $85 \%$ of the value expected if all reactants were consumed to form crystals. This suggests that the intermediate network contains most of the atoms which will later crystallise (Fig. 4a). Second, we noticed that the signal of the amorphous intermediate presents no detectable evolution between $6 \mathrm{~ms}$ and $37 \mathrm{~s}$, see SI 4. We can thus assume that upon crystallisation the scattering signal will correspond to a linear combination of purely amorphous and purely crystalline contributions. Based on this assumption, we extracted the crystal contribution to the SAXS signal (materials and methods, Eq. S5) and evaluated the volume fractions, diameters of gyration, volumes and number concentrations of crystalline primary grains and crystalline particles at all reaction times (Eqs S1, S7, S8 and S10, and Figs. 4a, $\mathrm{b}$ and $\mathrm{c})$. 

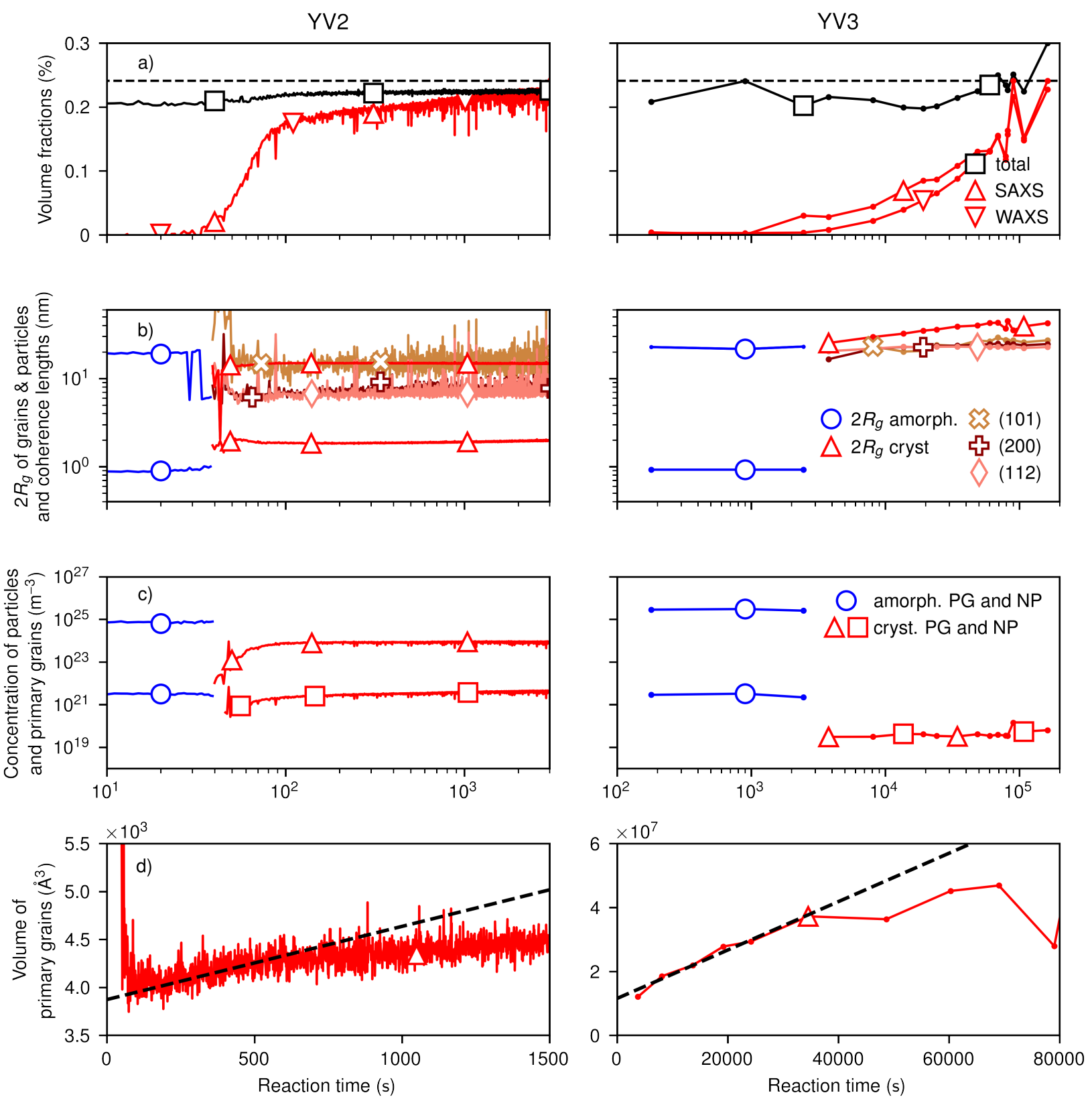

Figure 4: Physical parameters extracted from the SAXS/WAXS patterns, for the YV2 synthesis (left column) and the YV3 synthesis (right column). a) Volume fractions: total volume fractions expected for a complete reaction (----) , and measured from total scattering. Volume fractions from integration of the Bragg peaks, and from integration of the SAXS patterns. b) Sizes: diameters of gyration of the amorphous subunits, crystalline grains and particles, and coherence lengths from WAXS. c) Numeric concentrations of crystalline primary grains and particles. d) Volume of the primary grains. The dotted lines indicates the slopes used to calculate the growth rate $k_{\text {late }}$.

The SAXS contributions of the developing crystals unveil two different crystallisation 
scenarios depending on the initial $\mathrm{OH}^{-}$amount, $n$ (see Fig. 1). For $n=2$, nucleation results in the formation of crystalline primary grains with a diameter of gyration of $2 \mathrm{~nm}$, corresponding to the consumption of $c a$. 10 amorphous subunits within the pre-existing amorphous particle of $19 \mathrm{~nm}$ diameter of gyration. Regardless of the nucleation mechanism, as crystalline primary grains progressively increase in number within the amorphous particle (from $1 \times 10^{22}$ per $\mathrm{m}^{-3}$ of solution to $4 \times 10^{23} \mathrm{~m}^{-3}$ between 40 and $63 \mathrm{~s}$, Fig. $4 \mathrm{c}$ ), their probability of nucleation near an already formed crystalline primary grain increases. The crowding and the depletion of precursors inside the amorphous particle leads to aggregation of the crystalline primary grains, hence the formation of polycrystalline particles which progressively grow (Fig. 4b and Fig. 1), until depletion of reactants in the initial amorphous particle.

On the other hand, the monocrystals observed at $n=3$ appear not to form by rapid reconstruction of polycrystals. At intermediate crystallisation yields ( $\eta=20 \%$, Fig. 5$)$, direct inspection of the SAXS patterns evidence that the crystal contribution for $n=2$ presents a prominent correlation signal at $q \simeq 2 \times 10^{-1} \AA^{-1}$, characteristic of the accumulation of crystalline primary grains inside the polycrystalline particle. This signature is absent for $n=3$, evidencing that the crystalline nanoparticles at intermediate reaction times can be assimilated to single crystalline primary grains. The particles at $n=3$ thus form by nucleation of few single crystals growing across the whole amorphous particle and consuming ca. 37000 amorphous subunits instead of 10 for $n=2$, as depicted Fig 1.

The kinetic parameters measured at the nanometer-scale give insight on how the microstructures of the nanoparticles are determined by competition between nucleation and growth, combined with confinement in the a morphous $\mathrm{p}$ articles. When $n$ is increased from $n=2$ to $n=3$, the diameter of gyration of the crystalline primary grains grows from 2 $\mathrm{nm}$ to $\mathrm{ca}$. $30 \mathrm{~nm}$, suggesting that nucleation of new crystals becomes less favorable than growth on already formed crystals. We confirmed this a ssertion by comparing the kinetics of nucleation and growth : first, w e e stimated the s teady-state $\mathrm{n}$ ucleation $\mathrm{r}$ ates, $J$, from 

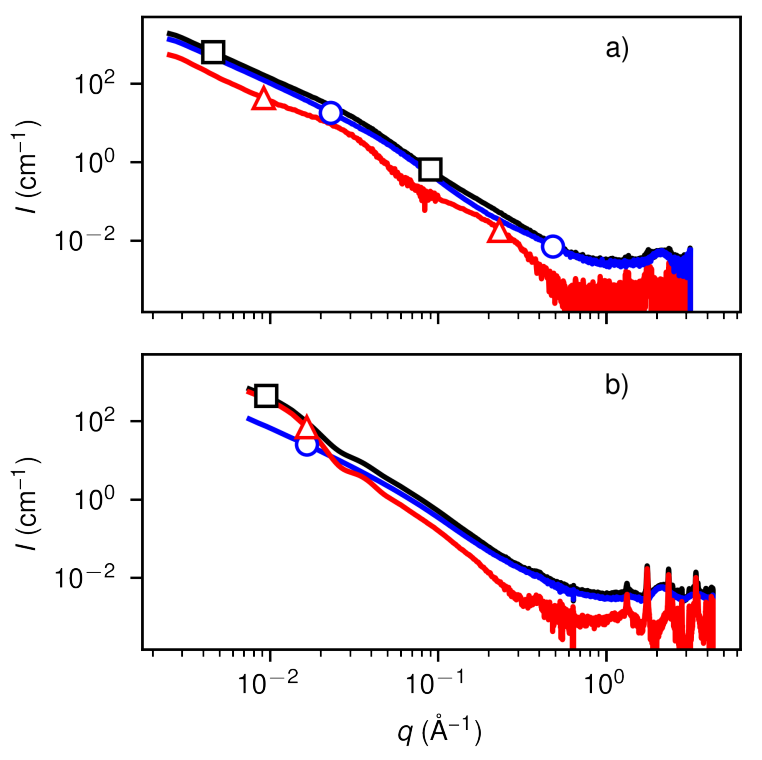

Figure 5: SAXS patterns taken at 20\% crystalline yield (- - ) and contribution of the crystals $\left(-\Delta_{-}\right)$and of the amorphous particles $(-\mathbf{-})$, a) for the YV2 synthesis $(t=48 \mathrm{~s})$ and $\left.\mathbf{b}\right)$ for the YV3 synthesis $(t=20 \mathrm{~min})$.

the evolution of the number of crystalline primary grains per unit volume (Fig. 4c). The steady-state nucleation rate, evaluated as the slope at early reaction times, decreases by seven orders of magnitude as $n$ varies from 2 to 3 (Table 2). Second, we retrieved two different mean growth rates (see schemes in Fig. 1): (i) the growth rate past the minimal observable size $k_{\text {late }}$ (Fig. $4 \mathrm{~d}$ ), which can be directly resolved from the increase in volume of the crystalline primary grains, and (ii) the growth rates of crystals immediately after nucleation $k_{\text {pop-corn }}$, from the critical nucleation size to the smallest observable size $(c a .1 \mathrm{~nm})$. Following Kaschchiev et al., $k_{\text {pop-corn }}$ is inferred from the induction time $t$, i.e. the time needed for a sufficient number of crystals to nucleate and grow until the lowest detectable crystal volume fraction $\phi_{m}=1 \times 10^{-4}$ :

$$
t=\left(\frac{2 \phi_{m}}{k_{\text {pop-corn }} V_{\mathrm{YVO}_{4}} J}\right)^{1 / 2}
$$

with $V_{\mathrm{YVO}_{4}}=80 \AA^{3}$ the volume of a $\mathrm{YVO}_{4}: \mathrm{Eu}$ formula unit.

We find that $k_{\text {pop-corn }}$ is at least fifty times higher than the growth rate at longer reaction 
times $k_{\text {late }}$ (Table 2). In the YV2 synthesis, crystalline primary grains grow from the critical size up to the detectable size at a rate of $c a . k_{\text {pop-corn }}(\mathrm{YV} 2)=0.4 \mathrm{~s}^{-1}$ to $8 \mathrm{~s}^{-1}$, then grow much slower at a rate $k_{\text {late }}(\mathrm{YV} 2)<1 \times 10^{-2} \mathrm{~s}^{-1}$. The decrease in the detected mean growth rate therefore suggests that the "pop-corn" nucleation/growth process prevails up to a particle size somewhere below the minimum detectable size, depletes the amorphous phase in reactants and hinders further growth at larger particle sizes. Similarly, in the YV3 synthesis, $k_{\text {pop-corn }}(\mathrm{YV} 3)$ is fifty times higher than $k_{\text {late }}(\mathrm{YV} 3)$, suggesting that once all reactants in the amorphous particle are used for the "pop-corn" growth, further growth is hindered.

In both cases the crystallisation reaction propagates within the amorphous particles across the nanometer-sized amorphous subunits via "pop-corn" nucleation/growth of crystalline primary grains, but growth above $c a .40 \mathrm{~nm}$ is precluded by depletion : a so-called "confinement e ffect". In addition, we observe that a high nucleation rate $\left(1 \times 10^{22} \mathrm{~m}^{-3} \mathrm{~s}^{-1}\right)$ leads to small primary grains $\left(8 \mathrm{~nm}^{3}\right)$ that form polycrystals, while a low nucleation rate $\left(1 \times 10^{15} \mathrm{~m}^{-3} \mathrm{~s}^{-1}\right)$ leads to much bigger primary grains $\left(c a .33500 \mathrm{~nm}^{3}\right)$ that become monocrystals. Polycrystallinity thus results from both (i) the competition between nucleation and growth and (ii) the confinement inside amorphous nanoparticles.

The confinement of $\mathrm{t}$ he $\mathrm{c}$ rystallisation $\mathrm{r}$ eaction i nside $\mathrm{t}$ he $\mathrm{v}$ olume of a morphous phase $V_{\text {amorph }}$ is also quantitatively supported by the relation between the nucleation and growth rates $J$ and $k_{\text {pop-corn }}$, and the number of crystalline primary grains after nucleation $N$. Assuming the reaction cannot consume more than a volume of metastable phase, $V_{\text {amorph }}$, we expect (see SI 6):

$$
N=V_{\text {amorph }} \sqrt{\frac{J}{k_{\text {pop-corn }} V_{\mathrm{YVO}_{4}}}}
$$

This is in good agreement with our experimental findings (Table 2): Eq. 2 combined with the experimental parameters yields $N(\mathrm{YV} 2)=32-143$ crystalline primary grains per particle, for $n=2$, against 180 experimentally measured. For $n=3$, we find that the time needed for a new crystal to nucleate inside an amorphous particle, $\left(J V_{\text {amorph }}\right)^{-1}$, greatly exceeds the time needed for an already formed crystal to consume the whole amorphous particle, 
$\left(k_{\text {pop-corn }} \frac{V_{\mathrm{YVO} 4}}{V_{\text {amorph }}}\right)^{-1}$, and measure accordingly $N(\mathrm{YV} 3)$ around unity.

The number of primary grains $N$ directly impacts the polycrystallinity (Fig. $4 \mathrm{~b}$, see also SI 5). For $n=3$, the crystal coherence length $(25 \mathrm{~nm})$ remains below the particle size (40 nm, corresponding to at most 4 crystallites per particle, depending on the hypotheses on their shapes and assuming no defects, Fig. 4b). In other words, the crystal coherence length for $n=3$ is roughly the size of a single amorphous particle, so YV3 nanoparticles can be considered monocrystalline. By contrast, for a $n=2$ synthesis, the crystal coherence length $(10 \mathrm{~nm})$ exceeds the size of the crystalline primary grains $(2 \mathrm{~nm})$, i.e. a crystal domain consists of $c a .75$ crystalline primary grains. A significant fraction of the crystalline primary grains are likely formed by secondary nucleation at the surface of already formed grains and extend the crystalline domains beyond the primary grains. However, because the amount of reactants is limited and several nucleation events occur, crystalline domains cannot extend to the whole amorphous particle, leading to polycrystals.

Our results demonstrate how the LaMer model must be adapted when a metastable, intermediate amorphous state of low solubility is involved. In that case, reactants first form dense amorphous particles of finites ize. C rystallisation then o ccurs c onfined in the amorphous particles. Therefore, the size of the amorphous intermediate particles governs the nanocrystal size; and the opposition between kinetics of nucleation and growth determine whether polycrystals or monocrystals are produced. In other words, the Lamer model is a poor indicator for the size of the nanoparticles, but instead becomes a reasonable guideline for the microstructure (primary grain size) of the nanoparticles, in an adapted version where the mother phase is the amorphous matrix instead of the solution. Our approach should provide efficient levers for synthesis control not only of chemical, but also of physical origin, as the structure of the amorphous phase seems independent on the chemical conditions. This in turn calls for a better understanding of the formation of the amorphous particles, here at reaction times lower than $6 \mathrm{~ms}$. 


\section{Acknowledgement}

We acknowledge a public grant overseen by the French National Research Agency (ANR), project DIAMONS ANR-14-CE08-0003. RKR acknowledges support by ANR as part of the "Investissements d'Avenir" program (Labex NanoSaclay, reference: ANR-10-LABX-0035). We acknowledge SOLEIL and ESRF for provision of synchrotron radiation facilities and we would like to thank Dr Javier Perez for assistance in using beamline SWING, and Dr Sylvain Prevost for assistance in using beamline ID02.

\section{Supporting Information Available}

Materials and methods, powder X-ray diffraction patterns, time-resolved luminescence and WAXS, SAXS at 6ms, XRD analysis and derivation of the number of primary grains from kinetic parameters.

\section{References}

(1) Kramer, H.; van Rosmalen, G. In Encyclopedia of Separation Science; Wilson, I. D., Ed.; Academic Press: Oxford, 2000; pp $64-84$.

(2) Cölfen, H.; Antonietti, M. Mesocrystals: Inorganic Superstructures Made by Highly Parallel Crystallization and Controlled Alignment. Angew. Chem. Int. Ed. 2005, 44, $5576-5591$.

(3) Gebauer, D.; Völkel, A.; Cölfen, H. Stable Prenucleation Calcium Carbonate Clusters. Science 2008, 322, 1819-1822.

(4) Vekilov, P. G. The Two-Step Mechanism of Nucleation of Crystals in Solution. Nanoscale 2010, 2, 2346. 
(5) Habraken, W. J. E. M. et al. Ion-Association Complexes Unite Classical and NonClassical Theories for the Biomimetic Nucleation of Calcium Phosphate. Nat. Commun. 2013, 4, 1507.

(6) De Yoreo, J. J.; Gilbert, P. U. P. A.; Sommerdijk, N. A. J. M.; Penn, R. L.; Whitelam, S.; Joester, D.; Zhang, H.; Rimer, J. D.; Navrotsky, A.; Banfield, J. F.; Wallace, A. F.; Michel, F. M.; Meldrum, F. C.; Colfen, H.; Dove, P. M. Crystallization by Particle Attachment in Synthetic, Biogenic, and Geologic Environments. Science 2015, 349, aaa6760.

(7) Stawski, T. M.; van Driessche, A. E.; Ossorio, M.; Diego Rodriguez-Blanco, J.; Besselink, R.; Benning, L. G. Formation of Calcium Sulfate through the Aggregation of Sub-3 Nanometre Primary Species. Nat. Commun. 2016, 7, 11177.

(8) Liu, Z.; Zhang, Z.; Wang, Z.; Jin, B.; Li, D.; Tao, J.; Tang, R.; De Yoreo, J. J. Shapepreserving amorphous-to-crystalline transformation of CaCO3 revealed by in situ TEM. Proceedings of the National Academy of Sciences 2020, 117, 3397-3404.

(9) Dey, A.; Bomans, P. H. H.; Müller, F. A.; Will, J.; Frederik, P. M.; de With, G.; Sommerdijk, N. A. J. M. The role of prenucleation clusters in surface-induced calcium phosphate crystallization. Nature Materials 2010, 9, 1010-1014.

(10) Baumgartner, J.; Dey, A.; Bomans, P. H. H.; Le Coadou, C.; Fratzl, P.; Sommerdijk, N. A. J. M.; Faivre, D. Nucleation and Growth of Magnetite from Solution. Nat. Mater. 2013, 12, 310-314.

(11) Baumgartner, J.; Ramamoorthy, R. K.; Freitas, A. P.; Neouze, M.-A.; Bennet, M.; Faivre, D.; Carriere, D. Self-Confined Nucleation of Iron Oxide Nanoparticles in a Nanostructured Amorphous Precursor. Nano Letters 2020, 20, 5001-5007, PMID: 32551668. 
(12) Besselink, R.; Stawski, T. M.; Freeman, H. M.; Hövelmann, J.; Tobler, D. J.; Benning, L. G. Mechanism of Saponite Crystallization from a Rapidly Formed Amorphous Intermediate. Crystal Growth \& Design 2020, 20, 3365-3373.

(13) Mirabello, G.; Ianiro, A.; Bomans, P. H. H.; Yoda, T.; Arakaki, A.; Friedrich, H.; de With, G.; Sommerdijk, N. A. J. M. Crystallization by particle attachment is a colloidal assembly process. Nature Materials 2020, 19, 391-396.

(14) Smeets, P. J. M.; Finney, A. R.; Habraken, W. J. E. M.; Nudelman, F.; Heiner, F.; Laven, J.; DeYoreo, J. J.; Rodger, M.; Sommerdijk, N. A. J. M. A Classical View on Nonclassical Nucleation. PNAS 2017, 114, E7882-E7890.

(15) Fleury, B.; Neouze, M.-A.; Guigner, J.-M.; Menguy, N.; Spalla, O.; Gacoin, T.; Carrière, D. Amorphous to Crystal Conversion as a Mechanism Governing the Structure of Luminescent $\mathrm{YVO}_{4}$ :Eu Nanoparticles. ACS Nano 2014, 8, 2602-2608.

(16) Kashchiev, D. Nucleation; Elsevier, 2000.

(17) Casanova, D.; Bouzigues, C.; Nguyên, T.-L.; Ramodiharilafy, R. O.; Bouzhir-Sima, L.; Gacoin, T.; Boilot, J.-P.; Tharaux, P.-L.; Alexandrou, A. Single Europium-Doped Nanoparticles Measure Temporal Pattern of Reactive Oxygen Species Production inside Cells. Nat. Nanotechnol. 2009, 4, 581-585.

(18) Fleury, B.; Dantelle, G.; Darbe, S.; Boilot, J. P.; Gacoin, T. Transparent Coatings Made from Spray Deposited Colloidal Suspensions. Langmuir 2012, 28, 7639-7645.

(19) Neouze, M.-A.; Freitas, A. P.; Ramamoorthy, R.-K.; Mohammedi, R.; Larquet, E.; Tusseau-Nenez, S.; Carrière, D.; Gacoin, T. Toward a Chemical Control of Colloidal $\mathrm{YVO}_{4}$ Nanoparticles Microstructure. Langmuir 2020, 36, 9124-9131, PMID: 32672970.

(20) Huignard, A.; Gacoin, T.; Boilot, J.-P. Synthesis and Luminescence Properties of Colloidal $\mathrm{YVO}_{4}$ :Eu Phosphors. Chem. Mater. 2000, 12, 1090-1094. 
(21) Mialon, G.; Türkcan, S.; Alexandrou, A.; Gacoin, T.; Boilot, J.-P. New Insights into Size Effects in Luminescent Oxide Nanocrystals. J. Phys. Chem. C 2009, 113, 1869918706.

(22) Teixeira, J. Small-Angle Scattering by Fractal Systems. J. Appl. Crystallogr. 1988, 21, $781-785$.

(23) Beaucage, G. Small-Angle Scattering from Polymeric Mass Fractals of Arbitrary MassFractal Dimension. J. Appl. Crystallogr. 1996, 29, 134-146.

(24) Kashchiev, D.; van Rosmalen, G. M. Review: Nucleation in Solutions Revisited. Cryst. Res. Technol. 2003, 38, 555-574. 